\title{
Fuzzy logic approach on traffic control of a Diverging Diamond Interchange in real time
}

\author{
Katarina Kukić ${ }^{1, *}$ and Aleksandar Jovanović ${ }^{2}$ \\ ${ }^{1}$ Faculty of Transport and Traffic Engineering, Department of Theoretical and Applied Mathematics, \\ Vojvode Stepe 305, Belgrade, Serbia \\ ${ }^{2}$ Faculty for Engineering, Department of motor vehicles and motors, Sestre Janic 6, Kragujevac, \\ Serbia
}

\begin{abstract}
In the recent paper, authors have considered a problem of controlling an oversaturated intersection in real time and developed a mathematical model based on fuzzy logic. The model was tested on an oversaturated " $\mathrm{T}$ " intersection and we obtained solutions with less control delay in comparison to the "fixed time" model. First, we will present the basic concepts of those results. In the rest of the paper, we apply a similar technique to develop a model for controlling a diverging diamond interchange -a type of diamond interchange in which the two directions of traffic on the non-freeway road cross to the opposite side on both sides of the bridge at the freeway. This type of interchange was developed in the 1970s in France but since 2009. it has been implemented in many different locations, above all in the USA.
\end{abstract}

\section{Introduction}

Nowadays, even more than in the last few decades, the problem of traffic congestion is present throughout the world. In large cities, this problem affects various aspects of every day's life. The negative consequences of traffic congestion from the point of view of traffic engineers are primarily time losses by traffic participants. But, from an ecological point of view, one must consider the impact congestion has on environmental pollution and also from the economic point of view the impact of congestion on higher fuel consumption. The authorities in many districts propose many measures and strategies to reduce traffic congestion. Some of them include the control of the operation of traffic signals and when it is possible building new roads, such as DDI. In this paper, we present the control of the operation of the traffic signals on a new, innovative type of road.

A Diverging Diamond Interchange (DDI) is a relatively new concept of intersection with an alternative geometrical structure. This type of diamond interchange became very popular, particularly in the USA, because it reduces vehicles control delays. The DDI also improves traffic safety because of left turn movements haven't any conflicts. The basic disadvantages of this concept are the relatively high initial construction costs and the space required for this design.

\footnotetext{
*Corresponding author: k.mijailovic@sf.bg.ac.rs
} 
In the review of hitherto literature, some relevant papers can be found. In [1] authors showed that DDI have better performance in comparison with a conventional diamond interchange, like control delays and queue lengths, for both vehicles and pedestrians. In [2] authors were investigating the case of the separate intersection control mode, under different geometric and demand conditions. The DDI in Texas, for example, shows the benefits under this approach. The concept of dynamically reversing lines was proposed in [3]. Results obtained by Binary Mixed Integer Linear Programming (BMILP) reveals the benefits in capacity expansion of traffic lanes. Authors in [4] discuss five different signal phasing schemes which could be implemented on DDI. Each of them has some advantages and disadvantages under specific traffic pattern and geometric layout of DDI. A methodology for offsets optimization on a corridor including a DDI was proposed in [5]. For intersections including DDI, it has been shown that the proposed methodology provides a saving in travel time. Some aspects of traffic safety at DDI was discussed by the authors [6]. Crash prediction models were developed for DDI in Missouri. The study found that the DDI is safer than the conventional diamond signalized intersection.

A lack of methodology for controlling this type of intersections leaves space for further research. In this paper, we consider the problem of controlling an oversaturated DDI. A mathematical model for controlling an oversaturated DDI, based on the fuzzy logic system "Sugeno" type is developed. The criterion function represents the control delay of vehicles in a certain period of analysis. The proposed model has been compared with "fixed time" control.

We considered the case when the intersection is oversaturated in all flows ("full oversaturated intersection"). Such a case is possible in peak periods of traffic load.

This work is organized in the following way: after the introductory review and a brief review of some of the important papers in this field, the second chapter is devoted to setting up the problems and goals of this paper. The third chapter provides a methodology for solving a problem based on the fuzzy logic system. In the fourth chapter, the proposed approach was tested on a hypothetical DDI. An analytic approach was used to compare the results obtained by the classic approach and the methodology proposed in this paper. We use the adaptation of geometrically based formula for delays obtained by Akcelik. Chapter 5 is dedicated to concluding observations and directions for future research.

\section{Setting up problems and goals}

This paper treats the oversaturated DDI, in terms of vehicle control delay minimization. The problem of controlling an oversaturated intersection by fuzzy logic has already been considered. Authors in [7] proposed a fuzzy algorithm for intersection with two oversaturated flows in mutual conflict. When the intersection is overloaded in all streams, "fixed time" control is recommended as it provides the same signalling plans as classical detector control, see [8]. In the paper [9] we developed a fuzzy algorithm for "fully" oversaturated intersection. The results, obtained in "T" intersection with one line per approach, shows the possibility to reach better performance in comparison to recommended control mode.

The aim of this paper is to apply the similar methodology used in [9], but now on the oversaturated DDI. This approach will be challenging because of the complexity of DDI. Early research is obtained only on the simple "T" intersection. The starting hypothesis is that applying the same fuzzy algorithm, less value of vehicles control delay can be reached compared to the classical control strategy. An analytical approach, particularly developed in this purpose, was used as a comparison method. 


\section{Methodology}

In the next few lines, we briefly present the methodology of controlling isolated " $T$ " intersection we developed in the paper [9]. We developed a mathematical model for controlling an oversaturated intersection based on the fuzzy logic system "Sugeno" type. The fuzzy logic controller that we developed is based on two input and one output fuzzy set. Input fuzzy sets are $V_{a p}$ - the number of vehicles in a row that is served during the green phase (vehicles approaching) and $V_{q}$ - the number of vehicles in a row waiting for the green time of the next phase (vehicles queuing). The input fuzzy sets consist of three membership functions represented by Gaussian curves: the small, middle and large order of vehicles.

For controlling DDI we set the limit values for the number of vehicles in a row on 80 . That number is obtained for specific intersection in the dependence of the distance of the detector and the number of traffic lanes. More detailed, from the geometry of the test DDI presented on Figure 1, the maximal number for approaches A and D is obtained by setting detectors on the distance of 200 meters. Considering that average vehicle's length is 5 meters, in one lane maximal number of vehicles between stop lane and a detector is 40 , multiplied by numbers of lanes, which is 2 in the approaches $\mathrm{A}$ and $\mathrm{C}$ gives the limit value of 80 vehicles. Similar, in the approaches B and D, detectors are set on the distance of 100 meters, which gives the maximal number of 20 vehicles per lane. Again, multiplied by the number of lanes which is 4 in this case, gives the limit value of 80 vehicles.

Fixed time control, based on historical value of vehicle flows, optimizes cycles and splits (solutions). Such predefined solutions are implemented over a certain period of the day. The criterion function for optimization, most often, is the vehicle control delays. More details about "fixed time" optimization may be found in [10].

After simulation for the case of "T" intersection, we calculated delays based on the formulas we developed starting from Discrete minimal delay model obtained by Akçelik in [11]. Delays we obtained were less than $12.54 \%$ for the fuzzy logic control compared to fixed time control. That motivated us to adopt a developed methodology for controlling signal plans for oversaturated DDI.

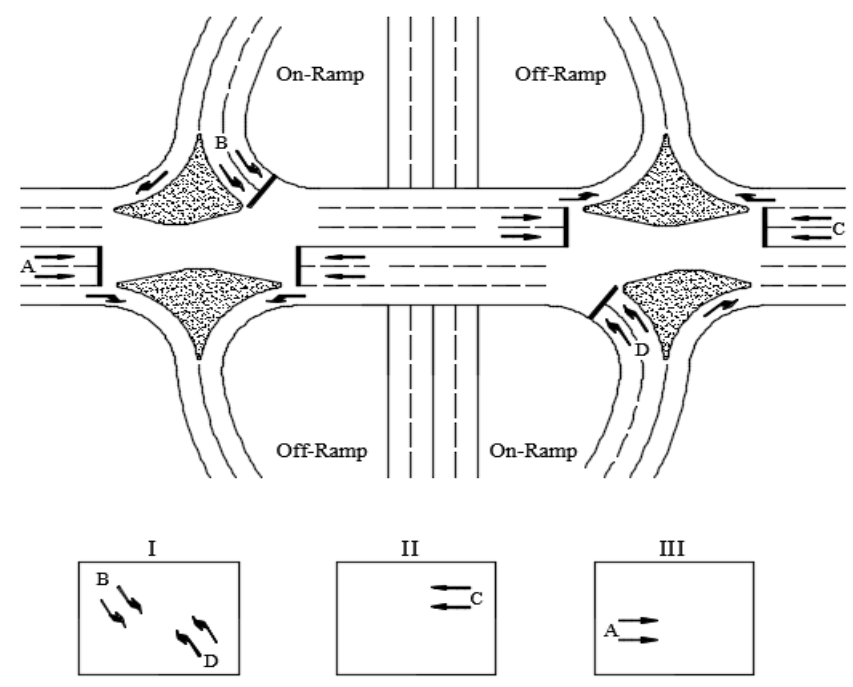

Fig. 1. Test DDI intersection

Most signal plans for DDI works in two phases, but in the situation of total oversaturation we didn't find it as a good choice, because cars from directions B and D 
would be prevented from entering the intersection by oversaturated directions $\mathrm{A}$ and $\mathrm{C}$. We suggest three phases as in Figure 1. The skipping of the phases is not allowed, nor the choice between the several phases to which will be assigned the green time. Input and output variables are the same as in previously explained " $T$ " intersection. The input variables are shown in Figure 2. and the algorithm for deciding on an extension or phase break is given in Figure 3.
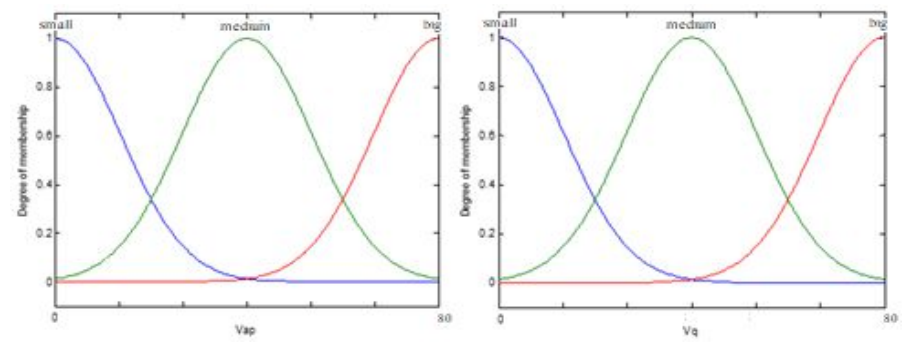

Fig. 2. The input variables into a fuzzy logical system

The fuzzy logic system has formed on the basis of the "If-Then" rules. "If" part of the rule is a premise, while "Then" part represents a consequence. In this case, "Then" part of the rule is a binary decision about whether to extend the phase for another two seconds (in phases two and three) or 6 seconds in phase one or to interrupt it. We extend phase one for 6 seconds because on approaches $\mathrm{B}$ and $\mathrm{D}$ we have left turns which takes more time than straight directions in approaches $\mathrm{A}$ and $\mathrm{C}$, which we extend for 2 seconds. The basis of the rule of the phase of the logical system is formed and shown in Table 1.

Table 1. Fuzzy rules base

\begin{tabular}{|c|c|c|c|c|}
\hline $\begin{array}{c}\text { Serial } \\
\text { number }\end{array}$ & $\begin{array}{c}\text { if Vap } \\
\text { (is }\end{array}$ & and Vq is & then EXT is & $\begin{array}{c}\text { Weight } \\
\text { factor }\end{array}$ \\
\hline 1. & small & small & 1 & 0.8 \\
\hline 2. & small & medium & 0 & 0.8 \\
\hline 3. & small & big & 0 & 1 \\
\hline 4. & medium & small & 1 & 1 \\
\hline 5. & medium & medium & 1 & 0.6 \\
\hline 6. & medium & big & 0 & 0.8 \\
\hline 7. & big & small & 1 & 1 \\
\hline 8. & big & medium & 1 & 0.8 \\
\hline 9. & big & big & 0 & 0.6 \\
\hline
\end{tabular}

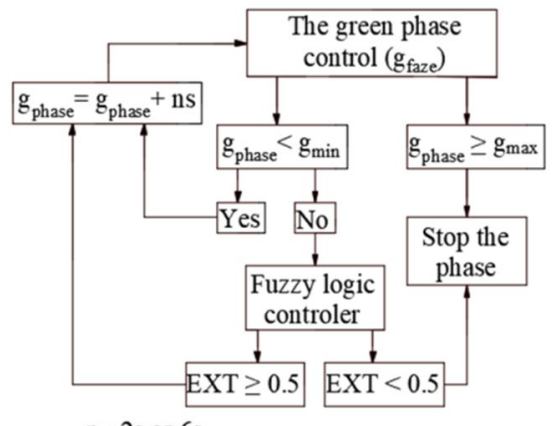

$\mathrm{n}=2 \mathrm{~s}$ or $6 \mathrm{~s}$

Fig. 3. The algorithm for control of the green phase time 
Each phase has a minimal green time $\left(g_{\min }\right)$ and maximal green time $\left(g_{\max }\right)$. Minimal green time is based on the distance of the detector from the stop line, while maximal green time is obtained from optimization in the "fixed time" control. The EXT value has a range between 0 and 1 . If the $E X T$ value is greater than or equal to 0.5 , the phase is extended by an extra 2 or 6 seconds (this choice will be explained later in the text); if less than 0.5 the current phase is stopped.

The values of the EXT and Weight factor are defined by the subjective opinions of the authors.

\section{Test example}

As a test example, we take a hypothetical DDI intersection with traffic flow in each approach having its own phase and with two traffic lanes at each approach. We located detectors in the approaches B and D on 100 meters from the stop line and in the approaches $\mathrm{A}$ and $\mathrm{C}$ on 200 meters from the stop line. In the simulation, we provided that vehicles are coming according to Poisson distribution. Our simulation process last 15 minutes.

On every $2 \mathrm{~s}$ the detector sends the binary information to the stage to a fuzzy logic controller in the form of 0 (no new vehicle) or 1 (precisely 1 vehicle appeared). We provide 2 seconds for serving the vehicle (when it leaves the stop line). By this setting, on every 2 seconds, the row of vehicles is updated at each of the intersection approaches, whether the queue has the green time or is waiting for service.

All red time during the cycle is 24 seconds. The red times (protection times) between phases one and two, and between phases three and one is $6 \mathrm{~s}$, while the red time between phases two and three is $12 \mathrm{~s}$. Within red phases, detector sends the information about new arrivals on the stop line. Such protection times are needed to avoid conflicts between vehicle queues when switching from one phase to another.

Based on the optimization model from Jovanović and Teodorović (2017) "fixed time" control is obtained. At the same time, the green timing of the phases obtained in this way represents the maximum green time in "real time" control. The solution gives a cycle value of $122 \mathrm{~s}$ and a green time value of $42 \mathrm{~s}, 28 \mathrm{~s}$ and $26 \mathrm{~s}$, respectively in phases. The minimum green time for all phases was adopted at $10 \mathrm{~s}$.

Fuzzy logic controller uses imbalances in the coming of the vehicle during the cycle. The solutions offered by the model developed in this paper are presented in the form: cycle length C; g1phase, g2phase, g3phase. The following solutions were obtained: 1) 94; 42, 18, 10. 2) $76 ; 18,18,16.3) 104 ; 30,30,20.4) 112 ; 42,28,18.5) 102 ; 42,10,26.6) 76 ; 12,30,10.7$ ) $68 ; 24,10,10.8) 56 ; 12,10,10.9) 56 ; 12,10,10.10) 56 ; 12,10,10.11) 56 ; 12,10,10$. In the last, the twelfth cycle, phase 3 was not tested because the analysis period, which was 15 minutes, expired.

Based on this outputs we calculated total delays both for the "fixed time" and for the fuzzy logic controller as a sum of all delays for seven cycles in the "fixed time" and for eleven cycles we obtained for the fuzzy logic control. For calculation of the delay we used Akçelik's discrete minimal delay model which we modified to work in three phases regime: 


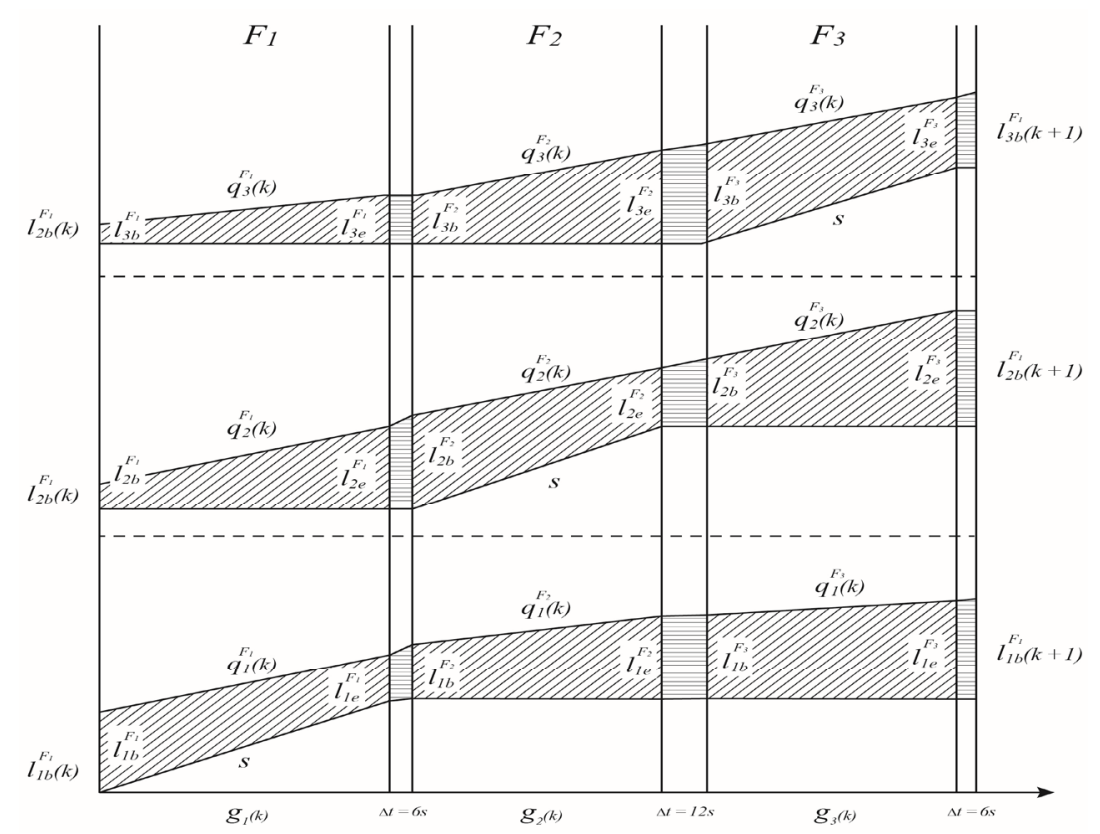

Fig. 4. The discrete minimal delay for three phases cycle

An explanation for the notation on the Fig. 4. is: $1_{1 \mathrm{~b}}(\mathrm{k})$ - queue length at the beginning of cycle state $\mathrm{k}$ with superscript $\mathrm{F}_{\mathrm{i}}$ denote the phase for which we calculate, similar $1_{\mathrm{le}}(\mathrm{k})$ denotes the queue length at the end of the observed phase. Further with $\mathrm{q}_{\mathrm{i}}(\mathrm{k})$ we denoted arrival flow rate for phase $\mathrm{i}, \mathrm{i}=1,2,3$ in the cycle $\mathrm{k}$. $\mathrm{C}$ is the cycle length, $\mathrm{s}$ is departure (saturation) flow rate and $g_{i}(k)$ is green time for phase $i$ in cycle $k$.

Finally, we obtain formulas for delays in the next form:

$$
\begin{gathered}
D_{1}^{F i}(k)=\left(l_{1 b}^{F i}(k)+l_{1 e}^{F i}(k)\right) \cdot \frac{g_{i}(k)}{2}, \quad \mathrm{i}=1,2,3 \ldots \\
D_{1 n i}^{F i}(k)=\left(l_{1 e}^{F i}(k)+l_{1 b}^{F i+1}(k)\right) \cdot \frac{d t_{i}}{2}, \quad \mathrm{i}=1,2,3 \ldots \\
\text { For } i=3: l_{1 b}^{F 3+1}(k)=l_{1 b}^{F 1}(k+1) \\
D_{1}(k)=D_{1}^{F 1}(k)+D_{1}^{F 2}(k)+D_{1}^{F 3}(k)+D_{1 n 1}^{F 1}(k)+D_{1 n 2}^{F 2}(k)+D_{1 n 3}^{F 3}(k) \\
D(k)=D_{1}(k)+D_{2}(k)+D_{3}(k) \\
D=\sum_{k} D(k)
\end{gathered}
$$

According to HCM (2010) saturation flow rate (s) can be expressed as 3600/headway $v e h / h$, where headway is average headway. During the "fixed time" optimization process, the value of saturation flows for straight movements (phases 2 and 3 ) and turn movements (phase 1) was adopted by $1800 \mathrm{veh} / \mathrm{h}$ and $1200 \mathrm{veh} / \mathrm{h}$, respectively. Because of that, in the proposed simulation, we consider the case when saturation flow rate $s$ is different in phase 
$\mathrm{F}_{1}$ compared with $s$ in $\mathrm{F}_{2}$ and $\mathrm{F}_{3}$. In phases two and three we considered $\mathrm{s}$ constant and equal to $1 / 2$ for each vehicle approach. In phase one we considered $s$ equal to $1 / 3$ (that explain ns $2 \mathrm{~s}$ or $6 \mathrm{~s}$ in figure 3 ). We calculated delays in Matlab, by using described methodology and formulas (1) - (6).

\subsection{Results}

We calculated control delays of vehicles in the case of fixed-time control (FTC) and also for the fuzzy logical time controller (FLTC) for the simulated data and with the values of green times, we presented at the beginning of this section. The values for 15 minutes of the simulation are shown in Figure 5.

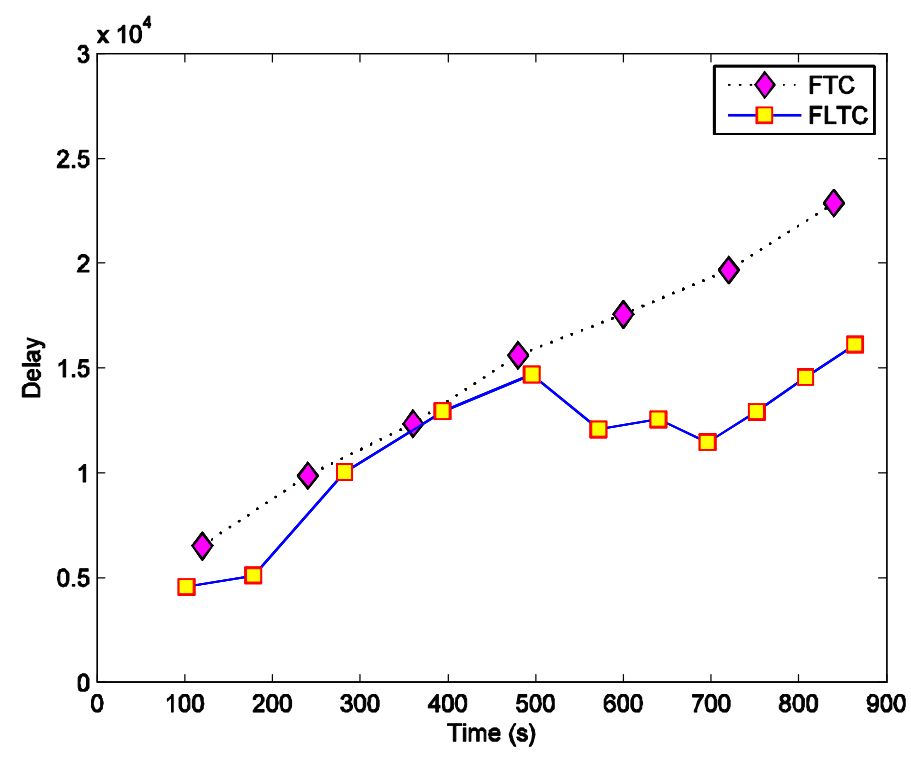

Fig. 5. The simulation results

After 15 minutes of simulation, the control delays of vehicles in the case of FTC control were 22864 s, while in the case of FLTC control were 17032 s. Fuzzy logic gave better results for $5832 \mathrm{~s}$ or $25.51 \%$ which is an even better improvement than in the case of " $\mathrm{T}$ " intersection where we obtained improvement of $12.54 \%$.

\subsection{Conclusions}

In this paper, we obtained real time controlling of fully saturated DDI. We implemented a similar methodology as in paper [9], expecting to obtain better results for fuzzy logic controlling than in the case of "fixed time" control. The results we obtained are even better than we expected and prove that it is possible to make a real time control of complex intersections such as DDI.

The authors are grateful to referees for usefull remarks. This research is supported by Serbian Ministry of Science and Technological Development, Project TR36002. 


\section{References}

1. P.K. Edara, J.G. Bared, R. Jagannathan, 3rd International Symposium on Highway Geometric Design, Chicago, IL. (2005)

2. R. Engelbrecht, K. Barnes, Journal of the Transportation Research Board, 1856, 231238. (2003)

3. J. Zhao, Y. Liu, X. Yang, Transportation research part C: emerging technologies, 51, 196-209. (2015)

4. Z. Tian, H. Xu, G. De Camp, M. Kyte, Y. Wang, 94th Annual Meeting of the Transportation Research Board, Washington, DC. (2015)

5. C. M. Day, S. M. Lavrenz, A.L. Stevens, R.E. Miller, D.M. Bullock, Transportation Research Record, (2016)

6. B. Claros, P. Edara, C. Sun, Accident Analysis \& Prevention, 100, 133-142. (2017)

7. L. Zhang, H. Li, P.D. Prevedouros, Transportation and Development Innovative Best Practices, 179-184 (2008)

8. R. P. Roess, E. Prassas, W.R. McShane, Traffic engineering, 4th ed. Upper Saddle River, N.J.: Prentice Hall. (2011)

9. A. Jovanović, K. Kukić, Military Technical Courier, 65, Issue 4, 866-881 (2017)

10. A. Jovanović, D. Teodorović, Transportation Planning and Technology, 40(5), 556576. (2017)

11. R. Akçelik, International Report AIR 367-1.Vermont South, Australia: Australian Road Research Board. (1980) 